\title{
Evaluating Predictors of Apple Scab with Receiver Operating Characteristic Curve Analysis
}

\author{
William W. Turechek and Wayne F. Wilcox
}

Cornell University, NYSAES, Department of Plant Pathology, Geneva, NY 14456.

Accepted for publication 2 February 2005.

ABSTRACT

\begin{abstract}
Turechek, W. W., and Wilcox, W. F. 2005. Evaluating predictors of apple scab with receiver operating characteristic curve analysis. Phytopathology 95:679-691.

Apple scab (Venturia inaequalis) is a perennial threat to apple production in temperate climates throughout the world. In the eastern United States, apple scab is managed almost exclusively through the regular application of fungicides. Management of the primary phase of disease is focused on preventing infection by ascospores. Management of secondary cycles of infection is largely dependent on how well primary infections were controlled. In this study, we used receiver operating characteristic curve analysis to evaluate how well mid-season assessments of the incidence of apple scab on cluster leaves, clusters (i.e., the whorl of cluster leaves), or immature fruit can serve as predictors of apple scab on har-
\end{abstract}

vested fruit (harvest scab) and whether these mid-season assessments of scab could be used reliably to manage scab under various damage thresholds. Results showed that assessment of scab on immature fruit was superior at predicting harvest scab than were assessments made on clusters or cluster leaves at all damage thresholds evaluated. A management action threshold of 7\% scab incidence on immature fruit was identified by Youden's index as the optimal action threshold to prevent harvest scab incidence from exceeding 5\%. Action thresholds could be higher or lower than $7 \%$ when economic assumptions were factored in to the decision process. The utility of such a predictor is discussed.

Additional keywords: Bayes theorem, disease management, risk management.
Apple scab (Venturia inaequalis) is a perennial threat to apple production in temperate climates throughout the world. The fungus is capable of attacking the foliage, blossoms, twigs, and most importantly, the fruit. The disease cycle of apple scab is characterized by two distinct but temporally overlapping spore stages. The primary stage is defined by the period of ascospore release. Venturia inaequalis overwinters as pseudothecia in fallen infected leaves, and ascospore maturity and release occur over a time period generally beginning at bud break in early spring and continuing until the ascospore supply is exhausted, which is soon after the completion of bloom. The peak release of ascospores typically occurs shortly before or during bloom. The secondary stage corresponds with the production of conidia and results in the classic polycyclic spread of disease. The first conidia are produced 2 to 4 weeks after the initial ascospore infections have completed their latent period and are found almost exclusively on the cluster leaves. Although these primary infections are visible, they are often difficult to find in commercial orchards because of their low number. Generally, detectable levels of apple scab are not present until shortly after petal fall or early- to mid-June in the Great Lakes Region of the United States.

In the eastern United States, apple scab is managed primarily through the application of fungicides (26). Protective sprays are applied on a calendar schedule or in response to Mills' table predictions $(17,19)$ beginning at bud break and continuing until 2 weeks after petal fall, generally an 8- to 10 -week period that includes 7 to 10 routine applications. The goal of this program is to protect susceptible tissues from ascosporic infections under the premise that good control of primary scab infection reduces substantially the risk of secondary cycles of infection and permits

Corresponding author: W. W. Turechek; E-mail address: turechew@ba.ars.usda.gov

DOI: 10.1094/PHYTO-95-0679

(C) 2005 The American Phytopathological Society a more relaxed series of cover sprays through the remainder of the season, reducing the costs of disease management. Implicit in this approach is an understanding that the susceptibility of fruit to infection by $V$. inaequalis decreases significantly approximately 2 to 4 weeks after petal fall $(24,25)$. In theory, this a good practice to follow, but perfect scab control is never achieved. Once the ascospore supply is depleted, growers typically make the decision whether to relax their cover spray schedule on the basis of the "feeling" of whether adequate protection was achieved through primary scab season, and less often, through assessment of the level of infection that has occurred.

One reason scab management is approached in this manner is because the quantitative relationship between primary scab levels and the necessary intensity of cover spray programs to prevent secondary scab from developing is not well defined. Moreover, it is unclear what the best indicator of the incidence of fruit infection at harvest is (herein referred to as harvest scab). Early fruit symptoms are thought to be the result of conidial infections produced from lesions on cluster leaves. This implies that assessment of scab on cluster leaves should serve as a good predictor of harvest scab. However, sampling individual cluster leaves after petal fall is time consuming and can be difficult because cluster leaves are sometimes small and hard to locate as terminal shoots begin to grow. Assessing the entire cluster for scab (i.e., the whorl of cluster leaves serves as the sampling unit), as opposed to individual cluster leaves, could alleviate some of the difficulties associated with cluster leaf sampling. We will show that the proportion of clusters infected with scab is easily described as a function of the proportion of cluster leaves infected with scab. Sampling developing fruit is another option. Intuitively, there should be a direct relationship between the number of developing fruit infected early in the season and harvest scab. Yet, sampling developing fruit as an indicator of harvest scab is an option only if there is a means to substantially reduce further fruit infections or stop existing infections. 
Fungicide efficacy trials for apple scab have been conducted routinely for many years and have focused heavily on the management of primary scab. Typically, several disease assessments are made throughout the season. The first assessment is taken 2 to 4 weeks after petal fall and focuses on how well primary scab was controlled. At this point, primary scab is clearly visible on the cluster leaves and fruit are approximately 20 to $30 \mathrm{~mm}$ in diameter. A second rating is taken near harvest, often to verify that control of primary scab was maintained through a typical cover spray program. Some trials, however, focus on developing or modifying cover spray programs or examine season-long programs of a single product. In all cases, the data generated from efficacy trials are often precise, detailed, and cover the full range of disease incidence levels; therefore, they may be very useful for identifying indicators or predictors of harvest scab.

Receiver operator characteristic (ROC) curve analysis is a statistical procedure used to identify and evaluate predictors of variables, for example, the prediction of harvest scab $(9,12,18,23)$. The ROC curve provides information showing how often predictions are correct and provides a graphical method for evaluating and discriminating between different predictors or modifications of the same predictor. The methodology has recently been introduced in the field of plant pathology $(12,38)$ and has been used subsequently to evaluate a prediction model for sclerotinia stem rot in oilseed rape (29) and the performance of the MARYBLYT system for predicting fire blight infections $(5,6)$. ROC curve analysis not only facilitates the selection of suitable predictors, but the ROC curve itself provides a means for risk management $(11,37)$. Metz $(18)$ showed how the ROC curve can be used to select thresholds on the basis of the economics of disease management and an estimate of disease incidence. Specifically, the costs of correctly managing disease, the expected losses based on mismanagement, and a means to estimate disease prevalence or incidence are required.

The objectives of this research were threefold: i) using fungicide efficacy trial data, characterize the relationship between the incidence of harvest scab and mid-season assessments of the incidence of early fruit scab and cluster leaf scab; ii) determine which of these mid-season scab assessments is the best predictor of harvest scab with ROC curve analysis; and iii) using the ROC curves, identify incidence thresholds that minimize economic losses due to harvest scab for specified harvest scab damage thresholds.

\section{MATERIALS AND METHODS}

Data. Data were collected from fungicide efficacy trials performed over the 5-year period from 1996-2000 (30-36). Depending on the year, trials were conducted in either one or two adjacent orchards on the Darrow Research Farm of the New York State Agricultural Experiment Station in Seneca Castle, NY.
Orchard 1 consisted of apple cultivars 'McIntosh' and 'Cortland' grafted on MM.106 rootstock with trees of each cultivar planted in alternation within rows. However, scab data were collected only from the 'McIntosh' trees. Orchard 2 was a solid block of 'Cortland' on MM.106 rootstock. Both orchards were planted in 1975.

Fungicide treatments varied according to their objectives and details can be found in Wilcox et al. (30-36). In general, treatments emphasized control throughout the period of primary scab infections using conventional fungicides that are currently registered or close to being registered for use against apple scab. Fungicide treatments began at the green-tip growth stage and continued through 2 weeks after petal fall (i.e., 1st cover spray). These sprays were followed by an additional four to five cover sprays of benomyl plus captan $(420 \mathrm{~g} / \mathrm{ha}$ and $3.36 \mathrm{~kg} / \mathrm{ha}$, respectively) applied through the remainder of the season. All primary scab treatments were applied with a handgun at a pressure of $2,413 \mathrm{kPa}$, and the additional cover sprays were applied with an airblast sprayer in a volume of water approximately onethird of that necessary for runoff.

In any single trial, treatments were arranged in a randomized complete block design with four replications. Apple scab was assessed twice during the season in each trial. The first assessment was done approximately 2 weeks after the application of the first cover spray to evaluate how well the program controlled scab during the primary phase (Table 1). In this assessment, the incidence of scab on individual cluster leaves, or cluster leaf incidence, was determined by examining all leaves on 25 fruiting clusters per tree, or 100 clusters per treatment, for the presence of apple scab symptoms and calculating the proportion of cluster leaves with scab from the total number assessed. The incidence of clusters (the whorl of cluster leaves) with scab, or cluster incidence, was calculated by rating a cluster as diseased if any leaf within the whorl was diseased and calculating the proportion of diseased clusters from the total number assessed. An estimate of cluster leaf incidence was derived from the incidence of diseased clusters using

$$
\operatorname{CLL}\left(\tilde{p}_{\mathrm{clv}}\right)=-\ln (a)+(1-b) \cdot \ln \left[\hat{p}_{\mathrm{cls}}\left(1-\hat{p}_{\mathrm{cls}}\right)\right]+\operatorname{CLL}\left(\hat{p}_{\mathrm{cls}}\right)
$$

in which $\operatorname{CLL}(z)=\ln [-\ln (1-z)]$ is the complementary log-log transformation of $z, a$ and $b$ are parameters analogous to the intercept and slope parameters of the binary power law, and the tilde $(\sim)$ is used to denote that the estimate of $p_{\text {clv }}$ was derived from measurements at another spatial scale $(13,27)$. To fit this model, $\operatorname{CLL}\left(\hat{p}_{\text {cls }}\right)$ was subtracted from both sides of equation 1 , i.e., $\operatorname{CLL}\left(\hat{p}_{\text {cls }}\right)$ is an "offset" variable, and $\ln \left[\hat{p}_{\text {cls }}\left(1-\hat{p}_{\text {cls }}\right)\right]$ was treated as an independent variable with slope $(1-b)$ and intercept $-\ln (a)[8,28]$. The deviance, analogous to the residual sum of squares (SSE) for normal-theory regression, was calculated for

TABLE 1. Incidence (proportion) of apple scab on cluster leaves, clusters, early fruit, and harvested fruit (harvest scab) on 'McIntosh' or 'Cortland' apple in an experimental orchard in New York

\begin{tabular}{|c|c|c|c|c|c|c|c|c|c|c|c|c|}
\hline \multirow[b]{2}{*}{ Year } & \multirow[b]{2}{*}{$\mathrm{Or}^{\mathrm{b}}$} & \multicolumn{3}{|c|}{ Cluster leaves } & \multicolumn{2}{|c|}{ Clusters $^{\mathrm{a}}$} & \multicolumn{3}{|c|}{ Early fruit } & \multicolumn{3}{|c|}{ Harvest scab } \\
\hline & & Date $^{c}$ & $\operatorname{Trt}^{\mathrm{d}}$ & $\mathrm{UTC}^{\mathrm{d}}$ & Trt & UTC & Date & Trt & UTC & Date & Trt & UTC \\
\hline 1996 & 1 & 16 July & 0.07 & 0.29 & 0.32 & 0.83 & 16 July & 0.11 & 0.86 & 19 August & 0.12 & 0.85 \\
\hline 1997 & 1 & 27 June & 0.08 & 0.63 & 0.28 & 0.96 & 27 June & 0.18 & 0.92 & 18 August & 0.24 & 0.99 \\
\hline 1998 & 1 & 19 June & 0.21 & 0.77 & 0.63 & 1.00 & 15 June & 0.19 & 0.86 & 11 August & 0.30 & 1.00 \\
\hline 1999 & 1 & 25 June & 0.17 & 0.43 & 0.48 & 0.84 & 25 June & 0.08 & 0.38 & 1 August & 0.08 & 0.69 \\
\hline 2000 & 1 & 19-20 June & 0.08 & 0.90 & 0.26 & 1.00 & 19-21 June & 0.08 & 0.91 & 21-22 August & 0.13 & 0.99 \\
\hline
\end{tabular}

a A cluster is the whorl of cluster leaves; if any cluster leaf within a cluster is diseased then the cluster is considered diseased.

b Orchard $1=$ McIntosh/Cortland orchard (trees planted in alternation within row); Orchard 2 = Cortland orchard.

${ }^{\mathrm{c}}$ Italics indicate median sample date if readings were taken over three or more days.

${ }^{d} \mathrm{Trt}=$ the mean incidence of all fungicide treatments within the trial, these varied according to the trial objective and are outlined in Wilcox et al. (30-36); UTC = untreated check. 
this model. It should be noted that if $a$ and $b$ were estimated via the binary power law, no curve fitting of equation 1 is necessary; although the two approaches would not necessarily lead to exactly the same estimates of $a$ and $b$ (28).

Fruit (100 per tree or 400 per treatment) were assessed for the presence of apple scab when cluster leaf incidence was assessed and then again in late August, the latter serving as the harvest scab assessment. The proportion of diseased fruit was calculated from the total number of fruit assessed. Second- or third-order polynomial models were used to characterize the relationship between each of the predictors (i.e., assessments of the incidence of early fruit scab, cluster incidence, cluster leaf incidence, and the prediction of cluster leaf incidence) and harvest scab; the models were fit via regression analysis.

ROC curve analysis. The data (i.e., individual treatment disease ratings) were partitioned into two groups according to a predetermined, somewhat arbitrary damage threshold ( $\left.D_{\text {thresh }}\right)$ for harvest scab. The "cases", denoted as $D+$, were treatments in which the incidence of harvest scab was known to exceed the damage threshold. The "controls", denoted as $D$-, were treatments in which the incidence of harvest scab was equal to or less than the damage threshold. Four damage thresholds were chosen on the basis that apples are produced for one of two predominant markets; the intended market destination dictates how the apples are grown. Fresh-market apples are sold primarily to grocers through wholesale distributors and must be of a certain size and blemish free. In New York, growers producing 'McIntosh' and 'Cortland' apples for this market on MM.106 rootstock can expect an average marketable yield of approximately $25 \mathrm{t} / \mathrm{ha}$ valued at approximately $\$ 315 /$ t. Processing apples are used for pies, apple sauce, and canning. They can be smaller than the apples destined for the fresh market and imperfections, to some degree, are acceptable. In New York, growers producing processing apples can expect an average marketable yield of $35 \mathrm{t} / \mathrm{ha}$ valued at approximately $\$ 157.5 / \mathrm{t}$. Apples that are undersized or severely blemished can be sold for juice at approximately $\$ 105 /$ t. For the purpose of this analysis, we consider managing harvest scab to below $2.5 \%$ incidence (i.e., $p=0.025$ ) as a reasonable damage threshold for fresh-market fruit (although this is arguably high for some growers, $2.5 \%$ harvest scab incidence can be sorted so that the packout is virtually scab free). For processing fruit, damage thresholds of 5, 10, and $25 \%$ harvest scab incidence were used, the latter for example only. That is, four distinct data sets were produced by partitioning the data into cases $(D+)$ and controls $(D-)$ on the basis of the four values of $D_{\text {thresh }}$. No analyses were conducted with a damage threshold $<2.5 \%$ because only two data sets were classified as controls when the data were partitioned according to a damage threshold of $1 \%$.

Measurements of the incidence of apple scab on (i) developing fruit $\left(p_{\text {efr }}\right)$; (ii) clusters $\left(p_{\text {cls }}\right)$; (iii) cluster leaves $\left(p_{\text {clv }}\right)$; and (iv) the estimate of diseased cluster leaves $\left(\tilde{p}_{\text {clv }}\right)$ were evaluated as predictors of harvest scab with ROC curve analysis. Each predictor was evaluated for its ability to classify harvest scab $\left(p_{\mathrm{hs}}\right)$ as above or below the damage threshold $\left(D_{\text {thresh }}\right)$ by recording the number of cases and controls that were correctly classified as such for each integer value of the predictor $(T)$ over its range of values $(0 \%<T<100 \%)$. Treatments with predictor values exceeding the so-called cutpoint value (i.e., $T>T_{\mathrm{cp}}$ ) were classified as diseased $(T+)$ and those with predictor values equal to or below the cutpoint $\left(T \leq T_{\mathrm{cp}}\right)$ were classified as not diseased $(T-)$. (The term "cutpoint" is equivalent to a management action or decision threshold for disease but was chosen over the latter term to avoid confusion with the use of "damage threshold" to classify data sets into cases and controls). Classifications were made irrespective of actual disease status. In total, 16 ROC curves were generated: four predictors applied to each of the four data sets.

The "sensitivity" of the predictor or the true positive proportion (TPP) is the conditional probability $P(T+\mid D+)$ and was calculated by dividing the number of true positive decisions by the total number of cases. The "specificity" of the predictor or the true negative proportion (TNP) is the conditional probability $P(T-\mid D-)$ and was calculated by dividing the number of true negatives by the total number of controls. The false positive proportion (FPP) is the conditional probability $P\left(T+\mid D_{-}\right)$and is calculated as $1-$ specificity. Similarly, the false negative proportion (FNP) is the conditional probability $P(T-\mid D+)$ and is calculated as 1 - sensitivity (11).

ROC curves were created for each predictor as a plot of TPP (sensitivity) versus FPP (1 - specificity) by allowing the cutpoint $\left(T_{\mathrm{cp}}\right)$ to vary over the range of its possible values (i.e., 0 to $100 \%$ $(11,18,20)$. The area under the ROC curve (AUROC) and its standard error were calculated for each curve. The calculation of the standard error is based on the equivalency that the AUROC, when calculated by the trapezoidal rule, is identical to the MannWhitney U-statistic $(1,4)$. The distribution of the Mann-Whitney U-statistic tends toward normality as the sample size increases, permitting the derivation of a $z$ statistic and a test of the hypothesis that the AUROC is no different than the area under the line of "no discrimination", i.e., 0.5 .

To compare AUROCs, a chi-square statistic with degrees of freedom equal to $N-1$ was derived from the covariance and correlation matrices of the Mann-Whitney U-statistic (4). Pairwise comparisons between the predictor $p_{\text {efr }}$ and the three other predictors were made with this statistic at each $D_{\text {thresh }}$ (4). A threeway comparison of the predictor's $p_{\text {cls }}, p_{\text {clv }}$, and $\tilde{p}_{\text {clv }}$ was also done with this statistic at each $D_{\text {thresh. }}$. With the three-way comparison, it is not possible to determine which of the curves is different from the others when significant differences exist, only that at least one curve had a different AUROC than the others.

Selecting management action thresholds. The ROC curve is a graphical summary of the performance of a forecaster expressed as a plot of its sensitivity and specificity for each cutpoint. Disease management decisions, however, require the user to select a single management action threshold or cutpoint along the ROC curve. If the ROC curve passes through the point $(0,1)$, it shows that the forecaster has at least one cutpoint possessing optimal sensitivity and specificity characteristics; however, this is rarely found. If sensitivity and specificity are considered equally important, such that the goal is to minimize the overall error rate, then the cutpoint closest to the point $(0,1)$ is considered the optimal cutpoint (18). This cutpoint, $T_{\mathrm{J}}$, was identified for each of the ROC curves produced using Youden's index:

$$
J=\text { sensitivity }+ \text { specificity }-1
$$

In practice, growers do not consider sensitivity and specificity equally important. Growers opt to err on the side of greater sensitivity, generally preferring to apply an erroneous extra spray rather than miss a necessary spray. What growers find difficult to assess in the decision process are the overall costs associated with each of the two possible errors and how these costs weigh into the decision process. Recognizing that the ROC curve has the general form TPP $=f(\mathrm{FPP})$, Hughes and Madden (11) showed how this expression could be integrated into a simple cost function where its first derivative with respect to FPP can be used to identify the slope at the point on the ROC curve where the average management costs are minimized relative to the errors. Preserving the notation of Hughes and Madden (11), the first derivative is

$$
f^{\prime}(\mathrm{FPP})=\left(\frac{C_{\mathrm{FP}}-C_{\mathrm{TN}}}{C_{\mathrm{FN}}-C_{\mathrm{TP}}}\right) \cdot\left(\frac{1-P(D+)}{P(D+)}\right)
$$

in which $C_{\mathrm{TP}}, C_{\mathrm{FN}}, C_{\mathrm{FP}}$, and $C_{\mathrm{TN}}$ are the costs of a true positive, false negative, false positive, and true negative decisions, respectively, $P(D+)$ is the prevalence of disease and represents the probability of harvest scab exceeding $D_{\text {thresh }}$, and $P(D-)=1-P(D+)$. 
$\left(C_{\mathrm{FP}}-C_{\mathrm{TN}}\right) /\left(C_{\mathrm{FN}}-C_{\mathrm{TP}}\right)$ is referred to as the cost ratio $(\mathrm{CR})$ and $1-P(D+) / P(D+)$ is the inverse odds ratio of the prevalence of disease, i.e., a roundabout measure of disease pressure.

To find the point on the ROC curve with slope $f^{\prime}(\mathrm{FPP})$ requires that the ROC curve be expressed in a functional form. Lloyd (15), and later Hughes and Madden (11), showed that the following equation can describe a variety of convex curves:

$$
\mathrm{TPP}=f(\mathrm{FPP})=\left[1+\exp (-\Delta) \cdot\left(\mathrm{FPP}^{-\mu}-1\right)\right]^{-1 / \mu}
$$

The derivative of equation 4 is:

$$
f^{\prime}(\mathrm{FPP})=\left[1+\exp (-\Delta) \cdot \mathrm{FPP}^{-\mu}-\exp (-\Delta)\right]^{-(\mu+1) / \mu} \cdot \exp (-\Delta) \cdot \mathrm{FPP}^{-(\mu+1)}
$$

in which $\Delta$ and $\mu$ are parameters to be estimated and are used simply to describe the shape of the ROC curve. Equation 4 is easily fit using any method to fit nonlinear models such as PROC NLIN (15). Finally, FPP can be estimated by rearranging equation 5 to express FPP as a function of $f^{\prime}(\mathrm{FPP})$ and substituting the value of $f^{\prime}(\mathrm{FPP})$ from equation 3 into equation 6 (11). TPP is estimated by substituting FPP from equation 6 into equation 4 .

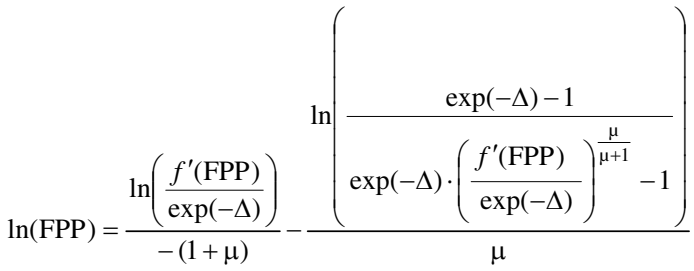

Equation 4 was fit to the data for each of the four predictors at each $D_{\text {thresh }}$ to estimate the parameters $\Delta$ and $\mu$ for the 16 ROC curves. Cutpoints corresponding to a cost ratio signifying a $5 \%$ crop loss for fresh-market apples $[\mathrm{CR}=(0.95 \cdot \$ 315+$ $0.05 \cdot \$ 105) / \$ 315]$ and processing apples $[\mathrm{CR}=(0.95 \cdot \$ 157.5+$ $0.05 \cdot \$ 105) / \$ 157.5]$, and a $100 \%$ loss of the crop to juicing for fresh market apples $(\mathrm{CR}=\$ 105 / \$ 315)$ and processing apples $(\mathrm{CR}=\$ 105 / \$ 157.5)$ at the prevalence values of $P(D+)=0.05$, $P(D+)=0.22$ and $P(D+)=0.75$ for each $D_{\text {thresh }}$ were identified using equations $4-6$. In calculating the cost ratio, we make the assumption that $C_{\mathrm{TP}}$ and $C_{\mathrm{TN}}$ are zero and that the cost of $C_{\mathrm{FP}}$ (i.e., an extra spray) is minimal relative to the loss in crop value. Thus, the cost ratio is simply the percent reduction in crop value due to scab for its intended market. The prevalence values $P(D+)=0.05$, $P(D+)=0.22$, and $P(D+)=0.75$ represent the probabilities that the incidence of harvest scab will not exceed 2,5 , and $28 \%$, respectively, and were calculated directly from the data. The values selected for $\mathrm{CR}$ and $P(D+)$ encompass a range of possibilities that growers are likely to realistically encounter.

Evaluating a predictor based on posterior probabilities. Sensitivity and specificity are two statistics useful for selecting optimal cutpoints. Their interpretation, however, is based on the condition of knowing if one is confronted with a case or a control. In practice, growers do not know this apriori. Indeed, what is of most interest to growers is the posterior probability of harvest scab exceeding $D_{\text {thresh }}$ given that the predictor has or has not exceeded $T_{\text {cp }}(11,37)$. Using an alternative formulation of Bayes' theorem, the posterior odds is defined:

$$
\operatorname{odds}(D+\mid T+)=\operatorname{odds}(D+) \times \operatorname{LR}(+)
$$

in which odds $(D+)=P(D+) /(1-P(D+))$ and $\mathrm{LR}(+)=$ TPP/FPP $=$ sensitivity/(1 - specificity) is the likelihood ratio of a positive prediction. Similarly, the odds of harvest scab exceeding $D_{\text {thresh }}$ given a negative prediction is:

$$
\operatorname{odds}(D+\mid T-)=\operatorname{odds}(D+) \times \operatorname{LR}(-)
$$

in which $\mathrm{LR}(-)=\mathrm{FNP} / \mathrm{TNP}=(1-$ sensitivity $) /$ specificity is the likelihood ratio of a negative prediction. It is clear from these expressions that the posterior odds of harvest scab exceeding $D_{\text {thresh }}$ is a function of the prior odds multiplied by the likelihood ratio. If $\operatorname{LR}(+)>1$, the posterior odds of harvest scab exceeding $D_{\text {thresh }}$ is greater than the prior odds subsequent to a positive prediction. Similarly, if $\operatorname{LR}(-)<1$, the posterior odds of harvest scab exceeding $D_{\text {thresh }}$ is less than the prior odds subsequent to a negative prediction. Thus, the best predictors are those in which $\mathrm{LR}(+)>1$ and $\operatorname{LR}(-)<1$ are met simultaneously.

The odds statement can be rearranged to express posterior probabilities using the relation $P(D \mid x)=\operatorname{odds}(D \mid x) /[\operatorname{odds}(D \mid x)+1]$. The probability $P(D+\mid T+)$ is known as the positive predictive value $(\mathrm{PV}+)$ and is a measure of the probability of harvest scab exceeding $D_{\text {thresh }}$ subsequent to a positive test result; $P(D-\mid T-)$ is the negative predictive value (PV-) and is a measure of the probability of harvest scab not exceeding $D_{\text {thresh }}$ subsequent to a negative test result. The latter is calculated as the complement of the posterior probability of disease given a negative test or 1 $P(D+\mid T-)$. For each predictor, the positive and negative predictive values were calculated for the cutpoints identified by minimizing equation 3 as described above.

The likelihood ratios and, by inference, the predictive values can be depicted graphically on the ROC plot and are a useful diagnostic when comparing cutpoints, or in the absence of an ROC curve (2). Specifically, for any select cutpoint, the slope of the line extending from the origin to the cutpoint is $\mathrm{LR}(+)$. The line with steepest slope has the highest $\operatorname{LR}(+)$ and the better $\mathrm{PV}(+)$. The line extending from the cutpoint to the point $(1,1)$ is LR(-). The line with the shallower slope has the highest LR(-) and $\mathrm{PV}(-)$.

Software. The statistical package GLIM was used to fit equation 1. AUROCs, standard errors, and all associated chisquare tests were calculated with the program AccuROC (version 2.4; Accumetric Corporation, Montreal, Canada). The SAS procedure PROC NLIN was used to fit equation 2. Calculations involving equations 2-10 were done in Excel (Microsoft, Redmond, WA).

\section{RESULTS}

The relationship between harvest scab incidence and each of the four predictors is shown in Figure 1. There did not appear to be any clear differences between treatments that relied on the standard cover spray program (open circles) and those that had cover sprays other than the standard (filled circles). The check (i.e., untreated) treatments were clearly responsible for "filling out" the graph at higher incidence levels and did not appear to deviate from the trend established by the treatments.

Early-season fruit scab was the most accurate predictor of harvest scab: $p_{\mathrm{hs}}=0.009+1.5 \cdot p_{\mathrm{efr}}-0.46 \cdot p_{\text {efr }}^{2}\left(R^{2}=0.93\right)($ Fig. $1 \mathrm{~A})$. It is particularly important to note that harvest scab was frequently higher than early fruit scab, indicating that additional fruit infections occurred after the first assessment and that a more intensive cover spray program could have reduced additional fruit scab symptoms. Cluster leaf scab and its prediction (based on fitting equation 1) were relatively poor predictors of harvest scab (Fig. 1C and D). Low levels of cluster leaf scab were often associated with relatively high levels of harvest scab, whereas the converse was also true at times. Cluster scab incidence levels, in contrast, were almost always higher than those for harvest scab (Fig. 1B). Unfortunately, the incidence and variability of cluster scab increased rapidly relative to increases in harvest scab incidence, making this an imprecise predictor. A cubic polynomial was fit to the data giving an $R^{2}$ of 0.74 .

The relationship between scab incidence on individual cluster leaves and whole clusters is shown in Figure 2. As expected, cluster incidence increased as a saturation-type curve with respect to cluster leaf incidence (13). Equation 1 provided a good fit to the data (solid line) with a deviance of $207(d f=144)$. The param- 
eter estimates and their standard errors (shown in parentheses) were: $-\ln (a)=1.108(0.0473)$ and $(1-b)=-0.1607$ (0.02109).

ROC curve analysis. Figure 3 shows the distribution of cases (lower half) and controls (upper half) depicted as two overlapping frequency distributions of cutpoint values for each predictor at each value of $D_{\text {thresh }}(23)$. Because the incidence of harvest scab was typically greater than $p=0.05$, the number of cases is greater than the number of controls when the data were partitioned by values of $D_{\text {thresh }} \leq 0.05$. Conversely, when $D_{\text {thresh }}=0.25$, there were more controls than cases. Ideally, the two distributions would not overlap and a cutpoint with perfect discrimination between cases and controls would lie between two nonoverlapping distributions. In our case, the two distributions never failed to overlap (Fig. 3). Thus, among the predictors and damage thresholds chosen, it is impossible to predict perfectly whether harvest scab would or would not exceed $D_{\text {thresh }}$. However, assessments of early fruit scab incidence $\left(p_{\text {efr }}\right)$ appeared to best discriminate between cases and controls (Fig. 3).

ROC curves are shown in Figures 4 and 5 and their corresponding AUROCs are listed in Table 2. In Figure 4, the ROC curves are grouped by the predictor, allowing comparisons among damage thresholds. In Figure 5, the ROC curves are grouped by $D_{\text {thresh }}$, allowing comparisons among predictors at each damage threshold. All AUROCs were significantly larger than 0.5 (i.e., the area under the line of 'no discrimination') according to MannWhitney U-statistic (Table 2). However, early fruit scab incidence $\left(p_{\text {efr }}\right)$ was clearly the best predictor of harvest scab incidence at every $D_{\text {thresh }}$. All AUROCs for early fruit scab incidence $\left(p_{\text {efr }}\right)$ were

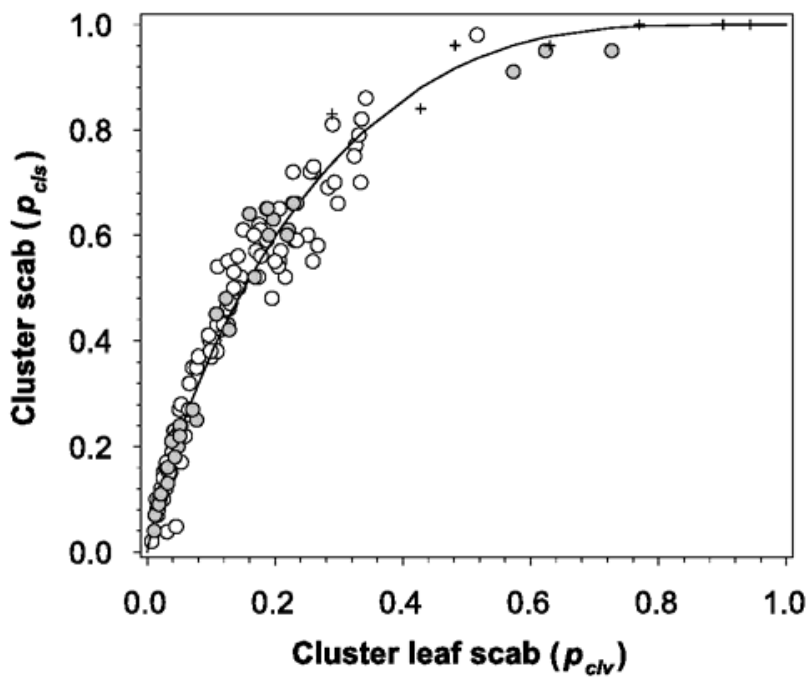

Fig. 2. Relationship between the incidence of apple scab on individual cluster leaves $\left(p_{\text {clv }}\right)$ and entire clusters $\left(p_{\text {cls }}\right)$ (i.e., the whorl of cluster leaves). The solid line represents the least squares fit of the data to equation 1; model parameters are given in the text. Open symbols represent treatments with a standard cover spray program, solid symbols represent treatments with a cover spray program other than the standard, and the crosses represent the untreated check.
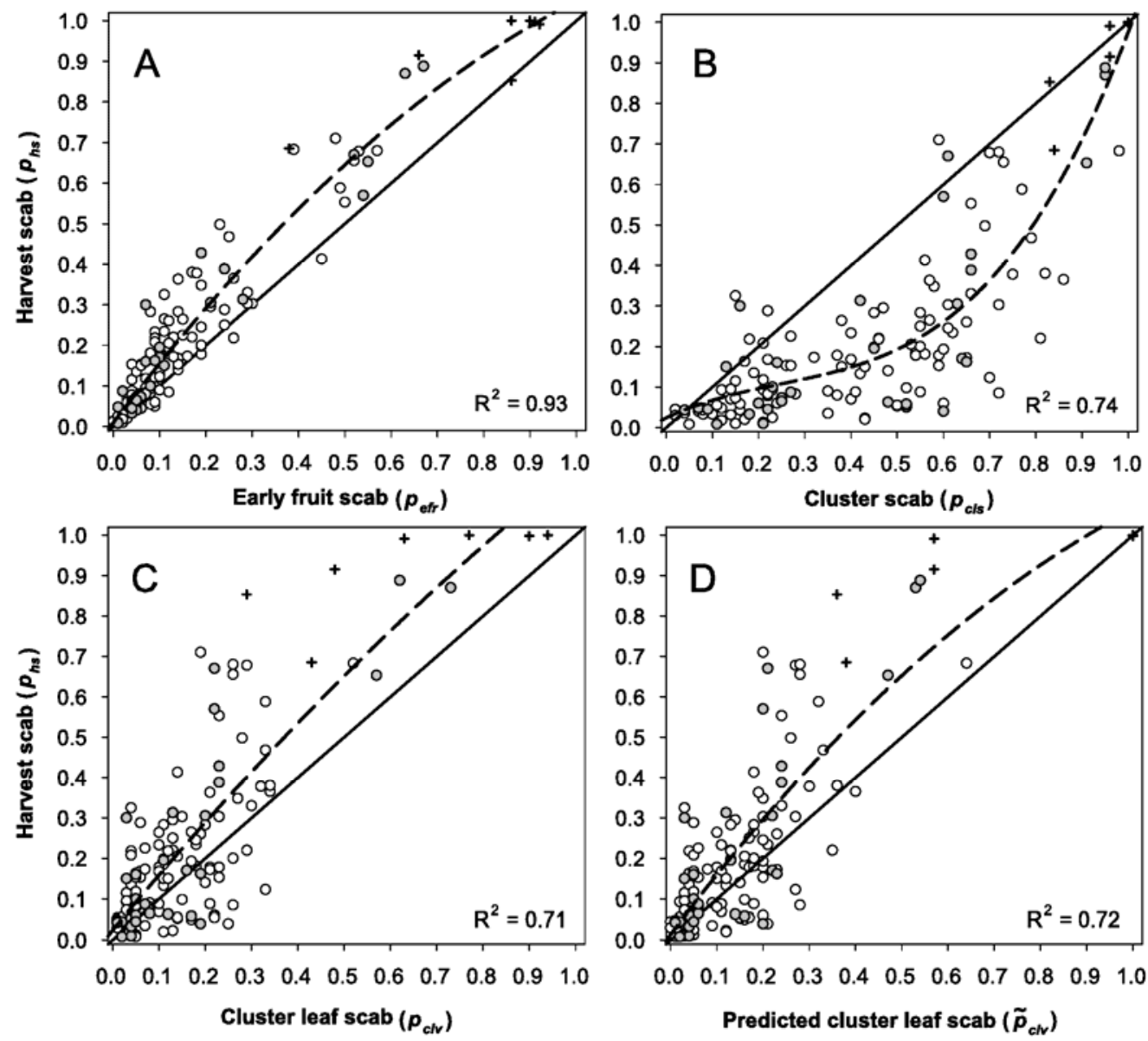

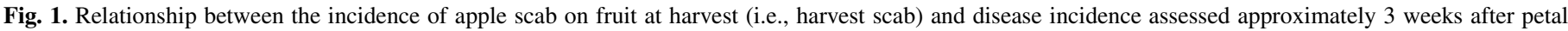

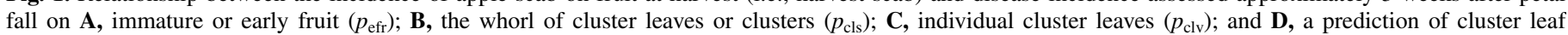

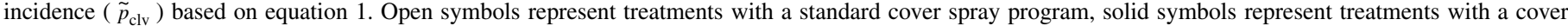

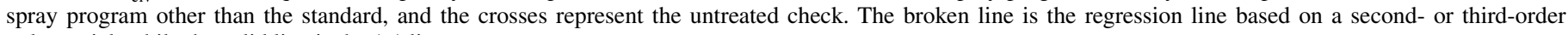
polynomial, while the solid line is the 1:1 line. 
$\geq 0.95$ and were significantly larger than the AUROCs for each of the other predictors $\left(p_{\mathrm{cls}}, p_{\mathrm{clv}}\right.$, and $\left.\tilde{p}_{\mathrm{clv}}\right)$ according to a chi-square test (Table 3 ). The AUROCs for $p_{\text {cls }}, p_{\text {clv }}$, and $\tilde{p}_{\text {clv }}$ were not significantly different from each other according to the three-way comparison (Table 3).

Selecting action thresholds. The optimal cutpoints identified by Youden's index $\left(T_{\mathrm{J}}\right)$ for each ROC curve are given in Table 2 and are shown as a vertical line for selected predictors in Figure 3 . As expected, $T_{\mathrm{J}}$ increased with increasing $D_{\text {thresh }}$ with the exception of $p_{\text {clv }}$ and $\tilde{p}_{\text {clv }}$ at $D_{\text {thresh }}=0.025$. For $p_{\text {efr }}, T_{\mathrm{J}}$ was higher than was $D_{\text {thresh }}$ at $D_{\text {thresh }}$ values of $p=0.025$ and $p=0.05$, but lower for

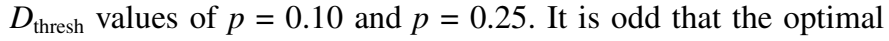
cutpoint for managing harvest scab based on assessments of early fruit scab is a value higher than the damage threshold (i.e., $T_{\mathrm{J}}>$ $\left.D_{\text {thresh }}\right)$. This could be explained by sampling error and/or may be the result of the preferential loss of diseased fruits during the natural thinning event known as "June drop", which would have occurred after the early fruit scab assessments were made.

Estimates of the parameters $\Delta$ and $\mu$ for each of the ROC curves in Figure 4 (or 5) are given in Table 4. ROC curves based on equation 4 were generated for the predictors $p_{\text {efr }}$ and $p_{\text {cls }}$ for $D_{\text {thresh }}$ of $p=0.05$ and $p=0.10$. The unique cutpoints were super-
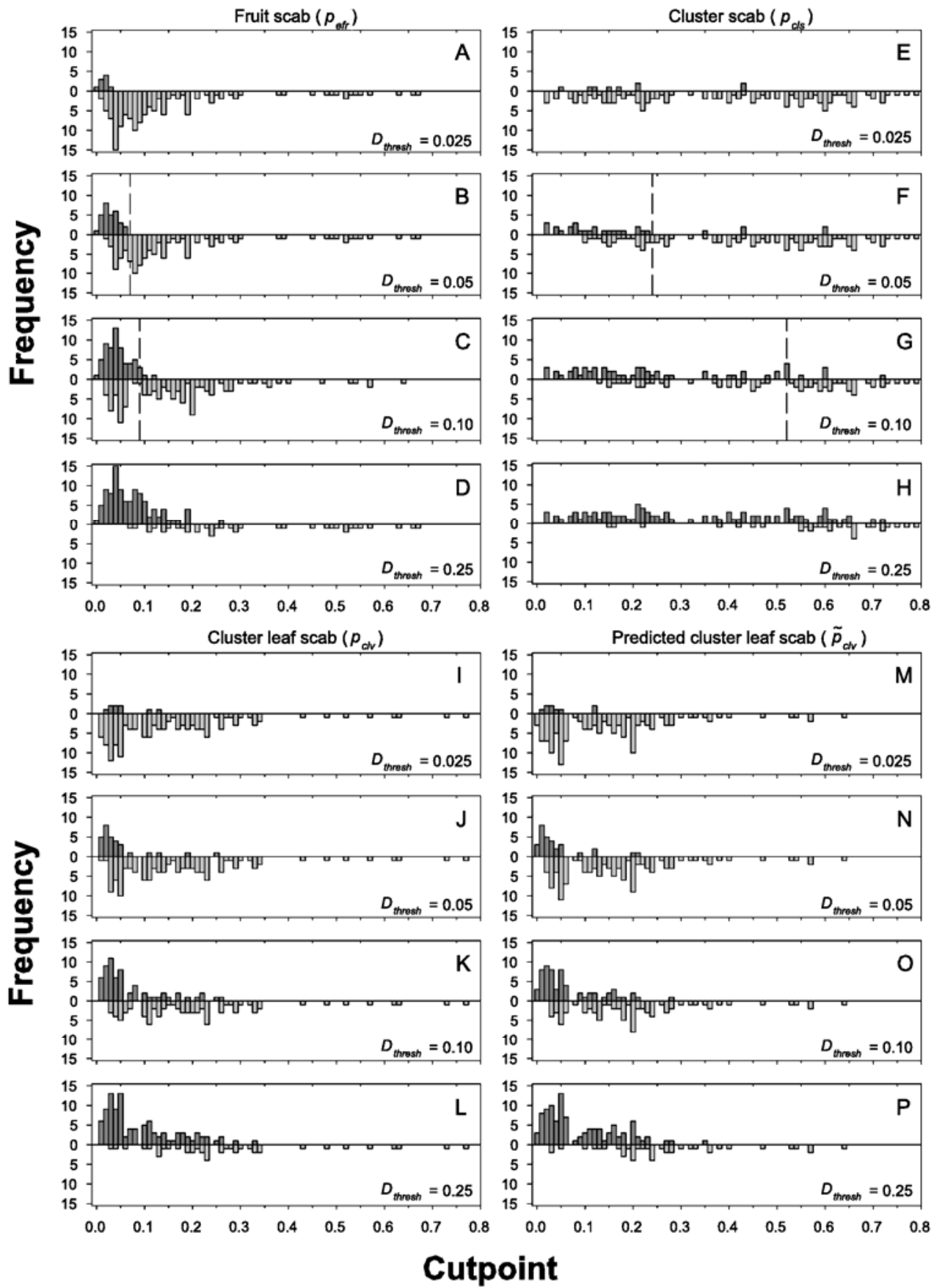

Fig. 3. The frequency of cases (lower histogram) and controls (upper histogram) over the distribution of management action thresholds (i.e., cutpoints) for four predictors of harvest scab at four damage thresholds $\left(D_{\text {thresh }}\right)$. Cases are defined as treatments in which the incidence of scab exceeded the damage threshold and controls are treatments in which the incidence of scab fell below the damage threshold. The damage threshold is an arbitrarily chosen incidence of scab on harvested fruit (harvest scab) used to partition the data into cases and controls. The predictors were evaluated at $D_{\text {thresh }}$ incidence values of $p=0.025,0.05,0.10$, and 0.25 . Assessments of the incidence of apple scab on $\mathbf{A}$ to $\mathbf{D}$, early or immature fruit $\left(p_{\text {eff }}\right)$; $\mathbf{E}$ to $\mathbf{H}$, clusters (i.e., the whorl of cluster leaves $\left[p_{\text {cls }}\right]$ ); $\mathbf{I}$ to $\mathbf{L}$, cluster leaves $\left(p_{\text {clv }}\right)$; and, $\mathbf{M}$ to $\mathbf{P}$, the prediction of cluster leaf incidence based on equation $1\left(\tilde{p}_{\text {clv }}\right)$ served as predictors of the incidence of harvest scab as above or below $D_{\text {thresh. }}$. The vertical line in $\mathbf{B}, \mathbf{C}, \mathbf{F}$, and $\mathbf{G}$ is the cutpoint identified by Youden's index. 
imposed on these curves and their values printed offset in Figure $6 \mathrm{~A}$ and $\mathrm{B}$. Figure $6 \mathrm{C}$ and $\mathrm{D}$ show select thresholds based on minimizing the cost function defined in equation 6 for the cost ratios and disease prevalences defined in Table 5. The thresholds identified by Youden's index are shown on these figures as a solid circle.

In general, cutpoints located in the upper right quadrant of the ROC plot lie in a more conservative region of the plot than the cutpoints in the lower left quadrant. That is, growers choosing to use cutpoints in this quadrant are more likely to initiate a management action because lower levels of disease trigger an action. Conversely, cutpoints nearest the origin are the least conservative cutpoints; growers are less likely to initiate a management action using cutpoints in this region of the curve. With this knowledge, various "what if" scenarios can be evaluated. For example, by holding the cost ratio constant (circular and diamond symbols), cutpoints associated with a higher $P(D+)$ tend toward the upper region of the ROC curve (Fig. 6C and D). Thus, as the probability or risk of disease increases, the costs associated with an unnecessary fungicide application outweigh the potential loss in crop value due to a lack of fungicide coverage. Similarly, for cutpoints assigned the same $P(D+)$ [the triangular symbols], it is the cutpoint associated with the lowest cost ratio that tends toward the upper region of the ROC curve. In other words, growers choosing to use this cutpoint believe (or know) that as the damage potential increases, the costs associated with a possibly unnecessary fungicide application, it outweighs the potential loss in crop value due to a lack in fungicide coverage.

Evaluating a predictor based on posterior probabilities. The positive and negative predictive values for each cutpoint depicted in Figure 6C and D are given in Table 5. Given the same disease prevalence (i.e., $P(D+))$, cutpoints approaching the origin along the ROC curve have a higher PV(+) but lower PV(-). That is, the likelihood that a positive prediction is a "true positive" prediction
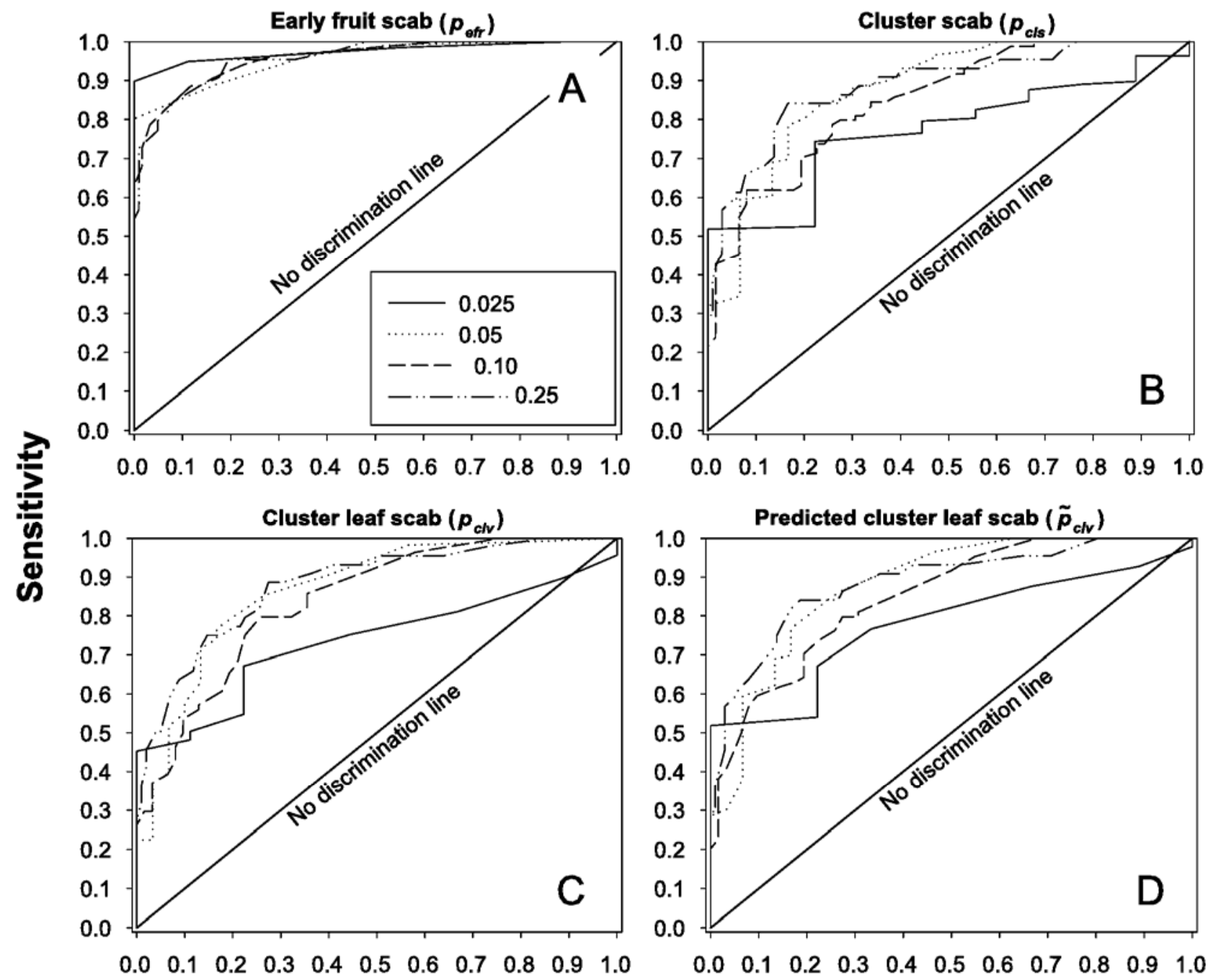

\section{1-Specificity}

Fig. 4. Receiver operating characteristic (ROC) curves for the harvest scab predictors $\mathbf{A}, p_{\mathrm{efr}}, \mathbf{B}, p_{\mathrm{cls}}, \mathbf{C}, p_{\mathrm{clv}}$, and $\mathbf{D}$, $\tilde{p}_{\mathrm{clv}}$ at damage thresholds $\left(D_{\mathrm{thresh}}\right)$ of $p=$ $0.025,0.05,0.10$, and 0.25 . The damage threshold is an arbitrarily chosen incidence $(p)$ of scab on harvested fruit (harvest scab) used to partition the data into cases (i.e., treatments in which harvest scab exceeded the damage threshold) and controls (i.e., treatments in which harvest scab did not exceed the damage threshold). The predictors in A to D, are mid-season assessments of the incidence of scab on developing fruit, clusters (i.e., the whorl of cluster leaves), cluster leaves, and a prediction of cluster leaf incidence based on equation 1, respectively. The false positive proportion (i.e., 1 - specificity), is the proportion of treatments that were incorrectly classified by the predictor as exceeding the damage threshold from the population of treatments that actually were below damage threshold (i.e., the number of controls incorrectly classified as a case). The true positive proportion, or the sensitivity, is the proportion of treatments that were correctly classified by the predictor as exceeding the damage threshold from the population of treatments that actually were above the damage threshold (i.e., the number of cases that were correctly classified as such). If the ROC curve falls along or below the line of no discrimination, the predictor does not predict better than chance. 
increases as cutpoints approach the origin, whereas the converse is true for negative predictions. Similarly, cutpoints approaching the point $(1,1)$ along the ROC curve have a higher PV(-) and lower PV(+); the likelihood that a positive prediction is a "true positive" prediction decreases as cutpoints approach the point $(1,1)$; the converse is true for negative predictions. One interpretation of the predictive values is as follows. Consider the point $\nabla$ in Table 5 under the damage threshold of 5\%. This corresponds to a management action threshold of approximately 8 to $9 \%$ scab incidence. Prior to assessing early fruit scab, the probability of harvest scab exceeding $5 \%$ is $22 \%$. Subsequent to a positive test, this probability increases to $86.1 \%$; subsequent to a negative test, this probability decreases to $100-93.4=6.6 \%$. Growers have the option of choosing cutpoints with higher or lower positive or negative predictive values depending upon their comfort for assuming risk. For example, consider Youden's index as a starting point. Growers who are willing to risk greater losses due to scab may choose a more liberal cutpoint located below Youden's index along the ROC curve or, conversely, will choose a more conservative one if they want to protect against greater losses.

The likelihood ratios for a positive and negative prediction are depicted graphically in Figure $6 \mathrm{E}$ and $\mathrm{F}$ for the cutpoint identified by Youden's index for $p_{\text {efr }}$ (broken lines) and $p_{\text {cls }}$ (solid lines) for $D_{\text {thresh }}$ of 0.05 and 0.10 . The line for $\operatorname{LR}(+)$ for the predictor $p_{\text {efr }}$ at $D_{\text {thresh }}=0.05$ is obscured because it falls along the $y$-axis. The ROC curves have been removed to reduce clutter, but the cutpoints listed in Table 5 have been retained for comparative purposes. In general, cutpoints located to the left of the $\mathrm{LR}(+)$ line have a superior $\mathrm{PV}(+)$ than does the cutpoint identified by Youden's index, cutpoints falling above the LR(-) line have a superior PV(-), and cutpoints meeting both criteria are overall superior to the cutpoint identified by Youden's index. For a given predictor, no one cutpoint is overall superior to the one identified by Youden's index, as expected. However, if the goal is to improve either $\mathrm{PV}(+)$ or $\mathrm{PV}(-)$, better cutpoints can be selected according to the criteria described. It should be noted that although the cutpoints shown in Figure 6 were selected according to conditions set in equation 3 , the same cutpoints can be arrived at in many ways, including finger pointing, and retain the same predictive properties.

\section{DISCUSSION}

The primary purpose of this research was to provide a means to classify correctly the incidence of harvest scab in an orchard block as above or below a specified damage threshold, rather than to provide an accurate estimate of harvest scab incidence. From a management perspective, it is often more useful to know whether disease will exceed some damage threshold than it is to have an estimate of absolute disease potential. The mid-season prediction of harvest scab will assist growers in determining whether to relax or intensify their cover spray program and will help them prior-

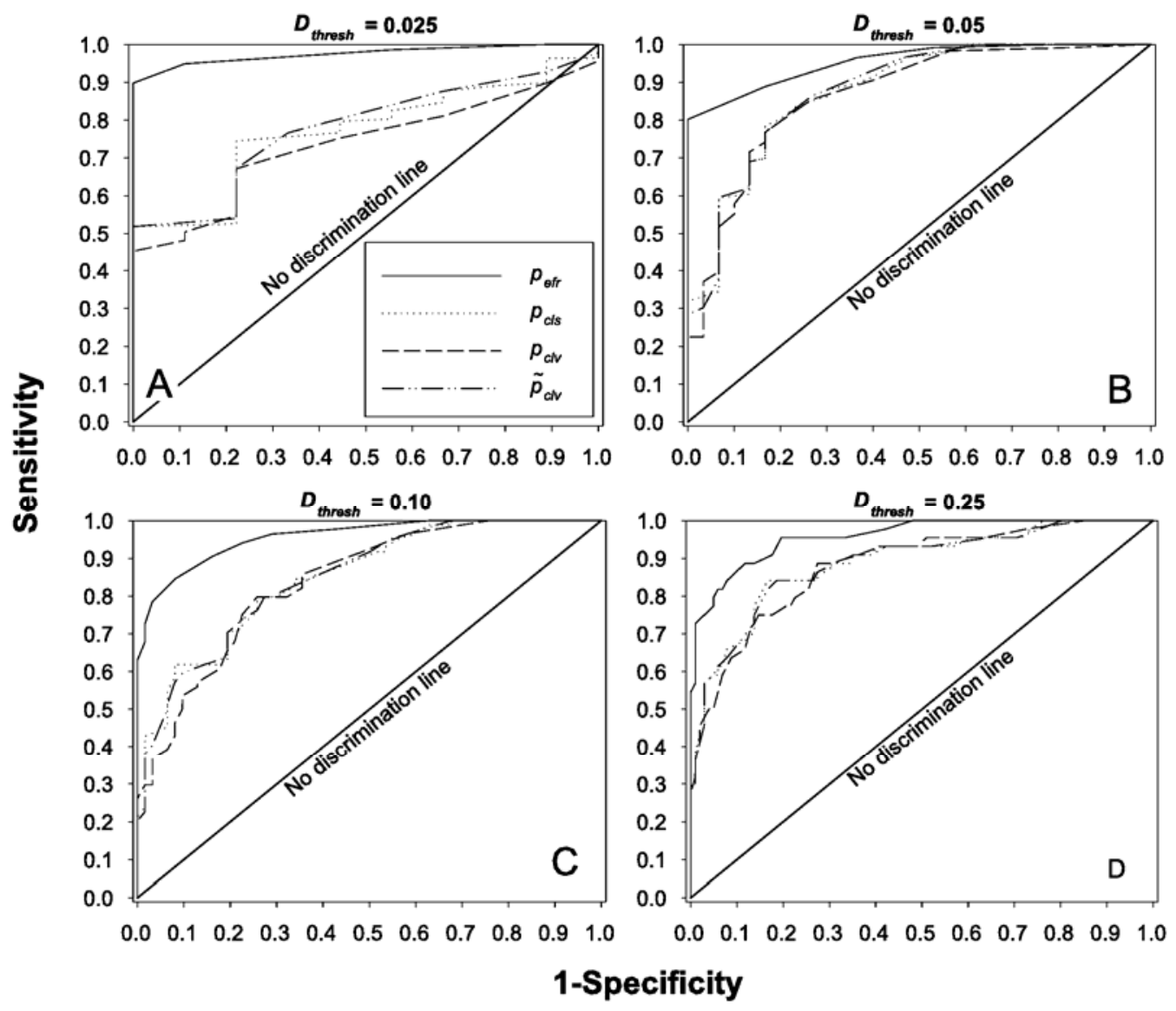

Fig. 5. Receiver operating characteristic (ROC) curves for the harvest scab predictors $p_{\text {efr }}, p_{\text {cls }}, p_{\text {clv }}$, and $\tilde{p}_{\text {clv }}$ at damage thresholds $\left(D_{\text {thresh }}\right)$ of $\mathbf{A}, p=0.025$, $\mathbf{B}, p=$ $0.05, \mathbf{C}, p=0.10$, and $\mathbf{D}, p=0.25$. The damage threshold is an arbitrarily chosen incidence $(p)$ of scab on harvested fruit (harvest scab) used to partition the data in to cases (i.e., treatments where harvest scab exceeded the damage threshold) and controls (i.e., treatments where harvest scab did not exceed the damage threshold). The predictors in each figure are mid-season assessments of the incidence of scab on developing fruit $\left(p_{\text {efr }}\right)$, clusters $\left(p_{\text {cls }}\right)$ (i.e., the whorl of cluster leaves), cluster leaves $\left(p_{\mathrm{clv}}\right)$, and a prediction of cluster leaf incidence based on equation $1\left(\tilde{p}_{\mathrm{clv}}\right)$. The false positive proportion (i.e., $1-$ specificity), is the proportion of treatments that were incorrectly classified by the predictor as exceeding the damage threshold from the population of treatments that actually were below damage threshold (i.e., the number of controls incorrectly classified as a case). The true positive proportion, or the sensitivity, is the proportion of treatments that were correctly classified by the predictor as exceeding the damage threshold from the population of treatments that actually were above the damage threshold (i.e., the number of cases that were correctly classified as such). If the ROC curve falls along or below the line of no discrimination, the predictor does not predict better than chance. 
itize the order in which orchard blocks should be sprayed. It is important to recognize that these predictors are intended to serve as part of a decision support system for managing harvest scab, irrespective of whether the support system is well-defined. That is, growers and advisors are expected to take the prediction results and consider them in terms of the susceptibility of the variety, the residual activity of previous and future fungicide applications, and the likelihood of another positive prediction in the near future, etc. Very few predictors or forecasters in use today serve as the sole basis for making decisions. Simply, forecasters are generally not designed to encompass the full variability in a typical agricultural system. It should be noted that the damage thresholds were chosen somewhat arbitrarily and represent benchmark figures. In practice, damage thresholds are variable and depend upon many factors including the economic conditions, the grower's financial standing, and the grower's production goals (21). A more thorough, and perhaps individualized, economic analysis is needed to determine if these thresholds are suitable for any single producer. Nonetheless, the lowest two thresholds likely fall into an acceptable range for growers of processing apples.
Relaxing a cover spray program is an option growers of processing fruit are more likely to embrace than growers producing fruit for the fresh market (e.g., growers producing fresh-market fruit assume that the incidence of harvest scab must fall below 1\%) (16). The choice to adjust a cover spray program based on a prediction of harvest scab implies: i) sampling was conducted at a time such that a reasonable prediction of harvest scab can be attained, ii) developing fruit are susceptible to infection, and iii) growers have options that will significantly alter the degree of harvest scab if mid-season thresholds were exceeded. With respect to the latter point, there are a number of fungicides on the market today with excellent protectant and postinfection activity against scab, which are effective at controlling scab when applied at virtually any point during the season. The first two points warrant further elaboration and will be covered in turn.

Addressing the issue of sampling, past research has shown that early-season assessments of foliar scab correlate poorly with the incidence and severity of harvest scab (16), findings that are supported here as well (Fig. 1). Even when assessed on the same date, it is clear that foliar scab ratings (i.e., cluster leaf and cluster

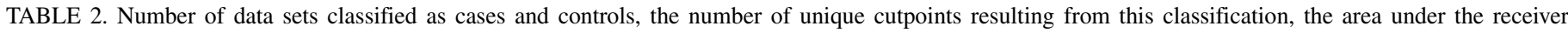
operating characteristic (ROC) curve with associated statistics, and the optimum cutpoint for the harvest scab predictors at specified damage thresholds

\begin{tabular}{|c|c|c|c|c|c|c|c|c|c|}
\hline Predictor $^{\mathrm{a}}$ & $D_{\text {thresh }}{ }^{\mathrm{b}}$ & Cases $^{c}$ & Controls $^{c}$ & Unique cutpoints ${ }^{\mathrm{d}}$ & AUROC $^{\mathrm{e}}$ & s.e. ${ }^{f}$ & $z^{\mathrm{g}}$ & $\operatorname{Pr}^{\mathrm{g}}$ & $T_{\mathrm{J}}^{\mathrm{h}}$ \\
\hline \multirow[t]{4}{*}{$p_{\text {efr }}$} & 0.025 & 137 & 9 & 46 & 0.975 & 0.012 & 4.77 & $<0.001$ & 0.04 \\
\hline & 0.05 & 116 & 30 & 46 & 0.958 & 0.015 & 7.68 & $<0.001$ & 0.07 \\
\hline & 0.10 & 84 & 62 & 46 & 0.957 & 0.014 & 9.44 & $<0.001$ & 0.09 \\
\hline & 0.25 & 44 & 102 & 46 & 0.958 & 0.016 & 8.77 & $<0.001$ & 0.11 \\
\hline \multirow[t]{4}{*}{$p_{\mathrm{cls}}$} & 0.025 & 137 & 9 & 70 & 0.770 & 0.058 & 2.71 & 0.003 & 0.22 \\
\hline & 0.05 & 116 & 30 & 70 & 0.878 & 0.037 & 6.37 & $<0.001$ & 0.24 \\
\hline & 0.10 & 84 & 62 & 70 & 0.849 & 0.031 & 7.20 & $<0.001$ & 0.53 \\
\hline & 0.25 & 44 & 102 & 70 & 0.887 & 0.031 & 7.41 & $<0.001$ & 0.55 \\
\hline \multirow[t]{4}{*}{$p_{\mathrm{clv}}$} & 0.025 & 137 & 9 & 41 & 0.735 & 0.585 & 2.36 & 0.009 & 0.14 \\
\hline & 0.05 & 116 & 30 & 41 & 0.867 & 0.384 & 6.20 & $<0.001$ & 0.06 \\
\hline & 0.10 & 84 & 62 & 41 & 0.839 & 0.324 & 6.95 & $<0.001$ & 0.10 \\
\hline & 0.25 & 44 & 102 & 41 & 0.879 & 0.031 & 7.27 & $<0.001$ & 0.13 \\
\hline \multirow{4}{*}{$\tilde{p}_{\text {clv }}$} & 0.025 & 137 & 9 & 40 & 0.777 & 0.058 & 2.79 & 0.003 & 0.13 \\
\hline & 0.05 & 116 & 30 & 40 & 0.879 & 0.037 & 6.40 & $<0.001$ & 0.06 \\
\hline & 0.10 & 84 & 62 & 40 & 0.849 & 0.031 & 7.20 & $<0.001$ & 0.10 \\
\hline & 0.25 & 44 & 102 & 40 & 0.886 & 0.032 & 7.40 & $<0.001$ & 0.17 \\
\hline
\end{tabular}

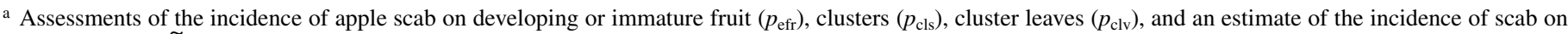
cluster leaves $\left(\tilde{p}_{\text {clv }}\right)$ (equation 1$)$ were evaluated as predictors of the incidence of harvest scab.

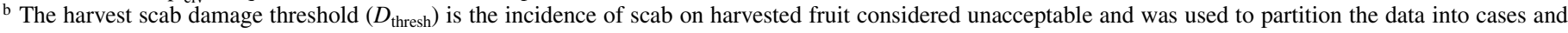
controls (the threshold is chosen arbitrarily).

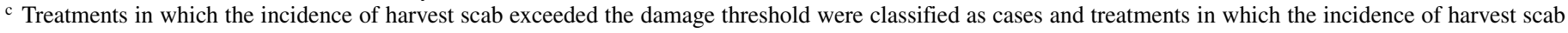
fell below the damage threshold were classified as controls.

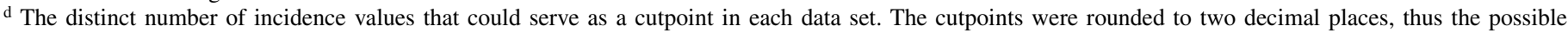
number of unique cutpoints for each predictor is 101 (i.e., $0,0.01,0.02 \ldots 1.0$ ).

e $\mathrm{AUROC}=$ area under the receiver operator characteristic curve.

f Standard error (s.e.) of the AUROC.

g Standard normal $z$-statistic and associated probability for testing the null hypothesis that AUROC $>0.5$.

h The optimal cutpoint identified by Youden's index (equation 2), i.e., the incidence value closest to the point $(0,1)$ on the ROC plot.

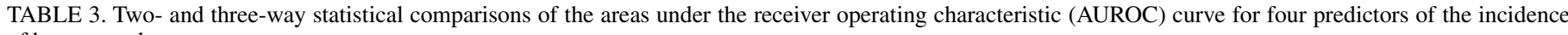
of harvest scab

\begin{tabular}{|c|c|c|c|c|c|c|c|c|}
\hline \multirow[b]{3}{*}{$D_{\text {thresh }^{\mathrm{c}}}$} & \multirow{2}{*}{\multicolumn{2}{|c|}{ Three-way test ${ }^{\mathrm{b}}$}} & \multicolumn{6}{|c|}{$p_{\text {efr }}{ }^{\mathrm{a}} \mathrm{Vs}$} \\
\hline & & & \multicolumn{2}{|c|}{$p_{\mathrm{cls}}$} & \multicolumn{2}{|c|}{$p_{\text {clv }}$} & \multicolumn{2}{|c|}{$\tilde{p}_{\text {clv }}$} \\
\hline & $\chi^{2}$ & $\operatorname{Pr}$ & $\chi^{2}$ & $\mathrm{Pr}$ & $\chi^{2}$ & $\operatorname{Pr}$ & $\chi^{2}$ & $\operatorname{Pr}$ \\
\hline 0.025 & 4.117 & 0.1276 & 14.409 & 0.0001 & 19.162 & 0.0000 & 13.350 & 0.0003 \\
\hline 0.05 & 2.059 & 0.3572 & 4.426 & 0.0354 & 5.274 & 0.0213 & 4.220 & 0.0400 \\
\hline 0.10 & 2.043 & 0.3600 & 16.932 & 0.0000 & 17.679 & 0.0000 & 17.046 & 0.0000 \\
\hline 0.25 & 0.598 & 0.7415 & 7.118 & 0.0076 & 9.109 & 0.0025 & 7.283 & 0.0070 \\
\hline
\end{tabular}

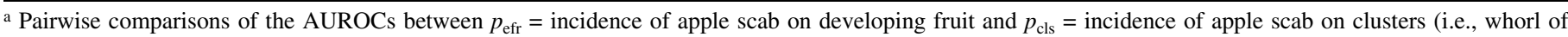
cluster leaves), $p_{\text {clv }}=$ incidence of apple scab on cluster leaves, and $\tilde{p}_{\text {clv }}=$ estimate of the incidence of scab on cluster leaves (equation 1 ). A chi-square statistic with 1 degree of freedom was calculated with the nonparametric method of DeLong et al. (4).

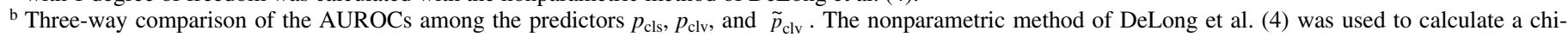
square statistic with 2 degrees of freedom.

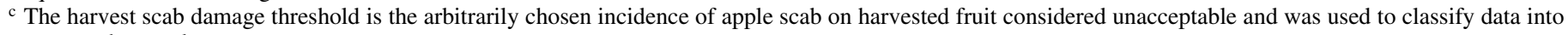
cases and controls. 
ratings) are inferior to ratings on early fruit for the prediction of harvest scab. This is attributed partially to the difficulty of detecting the disease on foliage when low incidence values exist. However, there are at least two practical approaches to help resolve this problem and expedite sampling. The first is sequential sampling. This is a common form of sampling often used by entomologists (3), but less so in plant pathology. The second approach is group or hierarchical sampling (13). This approach permits sampling at one hierarchical level-presumably the level easiest to collect information or one less prone to sampling error-and allows one to make inferences about the incidence of disease at a more relevant level. In this study, hierarchical sampling was used to make inferences of scab at the presumably more relevant level of the cluster leaves from assessments of cluster scab incidence (i.e., the upper level). Although neither cluster leaf incidence $\left(p_{\text {clv }}\right)$ nor its prediction $\left(\tilde{p}_{\mathrm{clv}}\right)$ were particularly good predictors of harvest scab, the fact that the two predictors had similar sensitivity (and specificity) suggests that hierarchical sampling could be used to classify an orchard block as above or below threshold presuming an adequate predictor.

The seasonal timing of sampling is also important because infections that have occurred within a minimum of 14 days of the assessment date will not be accounted for because of the long incubation period of apple scab. In western New York, late-June assessments of scab circumvent the lengthy incubation period because the peak susceptibility of cluster leaves, and fruit on some varieties, is at least 2 weeks past. To some degree, the issue of detection is also dealt with because all cluster leaf infections will be visible by this date and any significant amount of fruit infection should be detectable provided the sample size is reasonably large. Although it is possible that disease ratings made earlier or later in the season can lead to similar results, the mid-season rating is at least one window of opportunity where harvest scab can be reasonably predicted and the prediction is timely to allow the implementation of an intensified cover spray program to limit additional losses.

With this is mind, Linnet (14), when discussing the application of diagnostic tests under a clinical setting, stated "the diseased group should still be in an early phase of the disease, one at which it is still curable." In our setting, this relates directly to the susceptibility of fruit to secondary scab infections. Tomerlin and Jones (25) showed that beginning from approximately 3 weeks after petal fall through early August 'Jonathan' and 'Golden Delicious' fruit were highly resistant to infection even after exposure to $120 \mathrm{~h}$ of continuous wetness. 'McIntosh' apples, although exhibiting an increase in resistance during this period, were not only less resistant but became increasingly more susceptible to infection from late summer through the harvest period. Therefore, the results of this study may be indicative of a worst-case scenario for many varieties but a typical scenario for 'McIntosh', the leading variety grown in New York and New England. It is possible that thresholds could be scaled or adjusted according to variety, but it is not clear from this study how this could be accomplished.

A particularly relevant question is whether fungicide efficacy data were appropriate for developing thresholds for use in commercial orchards. The inoculum pressure is historically very high in the experimental orchards where the data were collected relative to most commercial orchards. This has advantages and disadvantages. The primary advantage is that the range of circumstances we wish to model occur in these orchard blocks; that is, primary scab develops to a degree in many treatments where a standard cover spray program fails to control secondary scab. Second, this data set encompasses the diversity of spray programs growers are likely to use for primary scab control. Third, any fungicide program that controls scab below threshold in these orchards will likely control scab in any commercial orchard. A disadvantage, however, is that disease pressure is quite high in these orchards making it extremely difficult to manage scab below a level acceptable for fresh-market fruit production. Consequently, the thresholds established here may not apply directly to fresh-market fruit production. Again, one may be able to "scale down" the results, but there are true differences in production practices that would argue against doing so.

ROC analysis was used to evaluate how well assessments of the incidence of early fruit scab, cluster scab, and cluster leaf scab taken 3 to 4 weeks after petal fall were at predicting the incidence of harvest scab as above or below various damage thresholds in a managed orchard. By comparing AUROCs, it was shown that assessment of early fruit scab was superior to assessments of incidence on clusters or cluster leaves for predicting harvest scab. However, conclusions drawn from analysis of the AUROCs should be considered with its limitations in mind. First, the AUROC curve represents "the average sensitivity obtained for a distribution of discrimination limits (cutpoints) giving a uniform distribution of specificities" (14). A complete range of specificities was represented in our data, thus lessening any concerns about the appropriateness of the analysis based on this factor. Second, the identity between the AUROC and the Mann-Whitney U-statistic implies that the AUROC is a measure of the probability that in randomly paired treatments, one from the cases and one from the controls, the predictor will classify them correctly $(1,9)$. Because of this, Hilden (10) makes the argument that the AUROC is a measure of detectability rather than a measure of diagnosticity. He further questions the validity of the statistic in a practical setting since it is rare that we are ever asked to decide which of two observations is the case and which is not.

Youden's index identified 7\% scab incidence on developing fruit and $24 \%$ incidence on clusters, our two select predictors, as the optimal cutpoints for managing harvest scab below a damage threshold of $5 \%$. When using Youden's index, it is assumed

TABLE 4. Parameter estimates from equation 4 for each predictor of the incidence of harvest scab at each damage threshold

\begin{tabular}{|c|c|c|c|c|c|}
\hline \multirow[b]{2}{*}{ Predictor $^{\mathrm{b}}$} & \multirow[b]{2}{*}{ Parameter $^{\mathrm{c}}$} & \multicolumn{4}{|c|}{$D_{\text {thresh }^{\mathrm{a}}}$} \\
\hline & & 0.025 & 0.05 & 0.10 & 0.25 \\
\hline \multirow{2}{*}{$\overline{p_{\text {efr }}}$} & $\mu$ & 0.0067 & 0.4850 & 0.4460 & 0.3551 \\
\hline & $\Delta$ & 3.7414 & 3.3606 & 3.4326 & 3.1830 \\
\hline \multirow{2}{*}{$p_{\mathrm{cls}}$} & $\mu$ & -0.5778 & 1.4320 & 0.3628 & 0.4467 \\
\hline & $\Delta$ & 0.9428 & 3.1092 & 1.8744 & 2.3675 \\
\hline \multirow[t]{2}{*}{$p_{\mathrm{clv}}$} & $\mu$ & -0.5039 & 1.2884 & 0.5369 & 0.3730 \\
\hline & $\Delta$ & 0.8299 & 2.9323 & 1.8004 & 2.1524 \\
\hline \multirow[t]{2}{*}{$\tilde{p}_{\mathrm{clv}}$} & $\mu$ & 0.5666 & 1.2251 & 0.3466 & 0.4889 \\
\hline & $\Delta$ & 1.4563 & 2.8852 & 1.7986 & 2.4401 \\
\hline
\end{tabular}

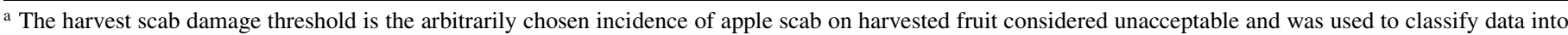
cases and controls.

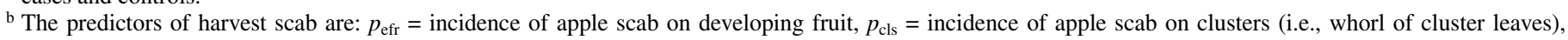
$p_{\text {clv }}=$ incidence of apple scab on cluster leaves, and $\tilde{p}_{\text {clv }}=$ estimate of the incidence of scab on cluster leaves (equation 1).

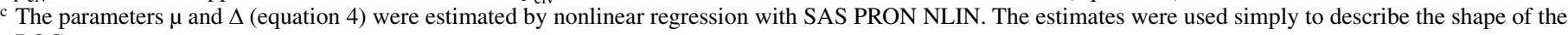
ROC curve. 
implicitly that sensitivity and specificity are equally important. However, growers do not consider sensitivity and specificity as equally important and are less likely to be concerned about an errant "extra" spray than a missed necessary spray. It is also assumed that disease prevalence, $P(D+)$, is a constant equal to the prevalence of cases in the data (i.e., $D+/ N$ ). Generally, disease prevalence is not constant. It varies according to climatic conditions and, in any single orchard, it is a function of varietal susceptibility and the disease history. The approach outlined by Metz (18) allows cutpoints to be chosen based on the user's comfort for assuming risk when the costs of false positive and negative decisions are known or can be assumed and/or an estimate of disease prevalence is available. This is the type of information given in Table 5 .

Fabre et al. (7) used this cost function to calculate the density of aphids on winter cereals warranting insecticidal treatment to prevent yield losses due to Barley yellow dwarf virus (BYDV). In their study, the data were partitioned into cases and controls based on a single damage threshold, and optimal decision thresholds were determined as a function of disease prevalence. As alluded

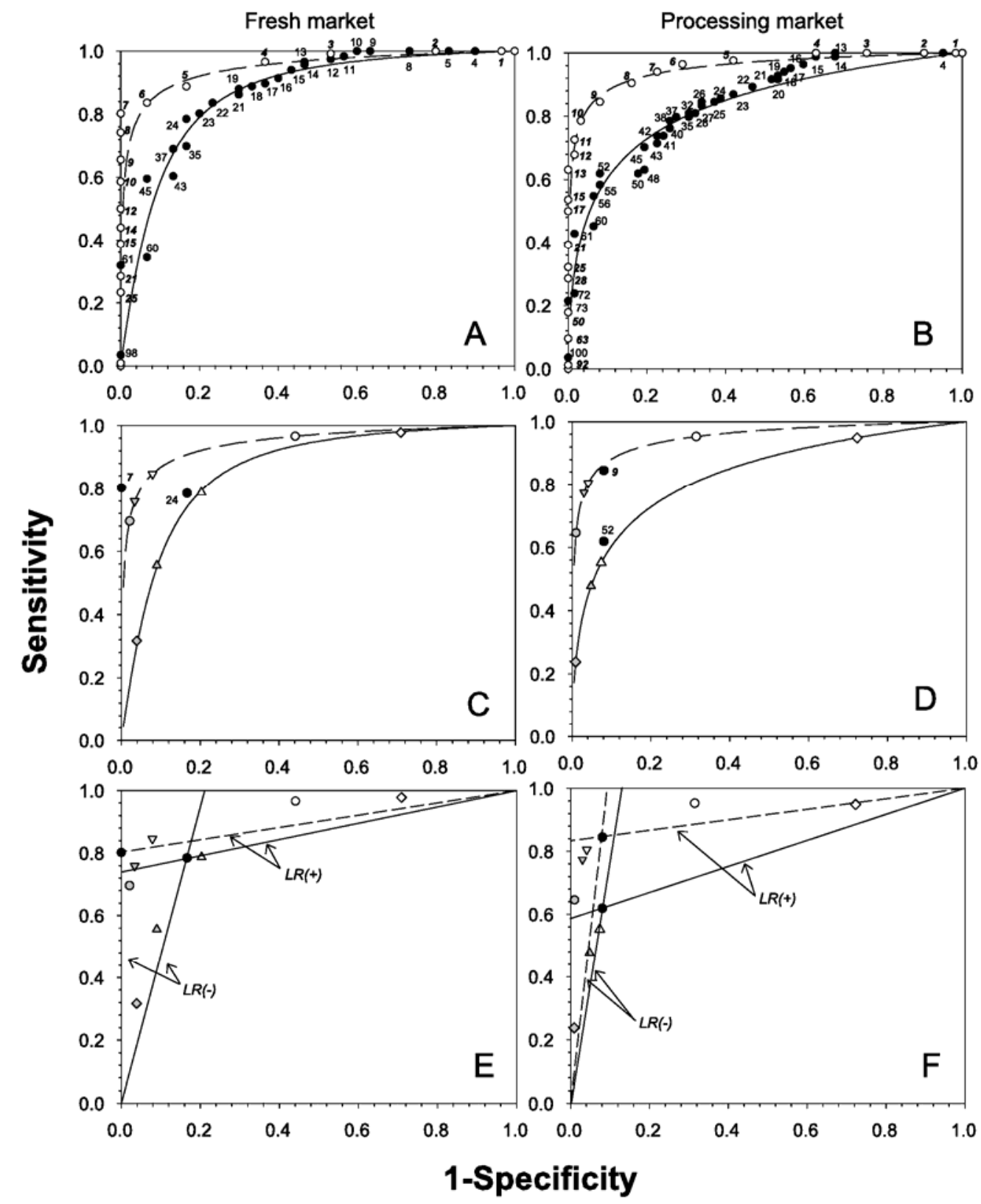

Fig. 6. A to D, Receiver operating characteristic (ROC) curves for the harvest scab predictors $p_{\text {efr }}$ (broken line) and $p_{\text {cls }}$ (solid line) at the damage thresholds $\left(D_{\text {thresh }}\right) \mathbf{A}, \mathbf{C}, p=0.05$ and $\mathbf{B}, \mathbf{D}, p=0.10$. The damage threshold is an arbitrarily chosen incidence $(p)$ of scab on harvested fruit (harvest scab) used to partition the data in to cases (i.e., treatments where harvest scab exceeded the damage threshold) and controls (i.e., treatments where harvest scab did not exceed the damage threshold). The predictors in each figure are mid-season assessments of the incidence of scab on developing fruit $\left(p_{\text {efr }}\right)$ and clusters $\left(p_{\mathrm{cls}}\right)$ (i.e., the whorl of cluster leaves). Select management action thresholds (cutpoints), based on the observed data, are superimposed on the curves in $\mathbf{A}$ and $\mathbf{B}$. Open symbols with bold, italicized labels represent ' $p_{\text {efr' }}$ ' cutpoints; solid symbols with standard labels represent ' $p_{\text {cls }}$ ' cutpoints. In $\mathbf{C}$ and $\mathbf{D}$, the labeled solid symbols are those cutpoints identified by Youden's index (equation 2). The remaining cutpoints have properties defined in Table 5. E, F, Graphical representation of the positive and negative likelihood ratios, $\mathrm{LR}(+)$ and $\mathrm{LR}(-)$, respectively, for the cutpoints identified by Youden's index for the harvest scab predictors $p_{\mathrm{efr}}$ (broken line) and $p_{\mathrm{cls}}$ (solid line). The remaining cutpoints are identical to those described in $\mathbf{C}$ and $\mathbf{D}$. The false positive proportion (i.e., 1 - specificity), is the proportion of treatments that were incorrectly classified by the predictor as exceeding the damage threshold from the population of treatments that actually were below damage threshold (i.e., the number of controls incorrectly classified as a case). The true positive proportion, or the sensitivity, is the proportion of treatments that were correctly classified by the predictor as exceeding the damage threshold from the population of treatments that actually were above the damage threshold (i.e., the number of cases that were correctly classified as such). 
to previously, the need for an estimate of disease prevalence is problematic because it has a large impact on the subsequent selection of management thresholds $(7,11,18)$ and the calculation of posterior probabilities $(10,11,37)$, but it is rarely known prior to when a decision must be made. Having settled on a predictor and damage threshold, Fabre et al. (7) calculated the frequency of correct and incorrect decisions over the range of decision thresholds (cutpoints), and the benefit of using this model was clearly demonstrated. This approach is recommended when a single predictor and damage threshold are chosen, and disease prevalence is expected to vary over a wide range. In our study, the selection of cutpoints was not the sole emphasis, but the choice of predictor as well as damage thresholds were also of considerable importance. Growers wishing to select specific management action thresholds could choose one selected in the study, select one by graphical approximation, or calculate a cutpoint by substituting their specific cost and disease prevalence values using the ROC curves presented here.

The choice to consider damage or yield loss as a binary variable instead of a quantitative one is problematic for at least two reasons. First, unless the economics of a system remain constant, damage thresholds are variable. But, if the degree of variability is relatively small over a reasonable course of time, selecting a single damage threshold, say the midpoint, seems appropriate. In our study, we tested four damage thresholds working under the assumption that we encompassed the range of damage thresholds acceptable to growers operating under different economic conditions. Selecting several thresholds is not necessarily the best approach. Hughes et al. (12), suggest plotting an ROC surface in which TPP and FPP are plotted over the possible range of damage thresholds. However, when one or a few suitable damage thresholds can be identified, plotting ROC curves is preferred because it makes explicit the implications of choosing a particular damage threshold when additional parameters affecting the outcome are investigated (12).

Second, dichotomizing the damage threshold to allow the classification of treatments into cases and controls is done under the assumption that the information derived from sampling is without error. This, of course, is not true. The uncertainty attached to the classification of treatments into case and control subgroups can be characterized by creating a sampling likelihood or an operating characteristic (OC) curve (12). Accounting explicitly for this error in the ROC analysis is not straightforward, but methods do exist (23). Thus, it is important that the partitioning of data into cases and controls be done with the greatest confidence possible because errors in the initial dichotomization due to the flawed sensitivity and/or specificity in the "gold standard" are carried through the analysis.

Ultimately, "the aim of performing a test is to move from the pretest disease probability $P$ to a post test probability $r$ that is hopefully near zero..., so that a safe therapeutic decision can be made" (10). This clearly provides motivation for using posterior probabilities to evaluate the performance of a predictor. The predictive capabilities of any number of cutpoints can be easily compared with a standard cutpoint, for example the one identified by Youden's index, by observing where they lie on the ROC plot relative to positive and negative ratio likelihood lines for the standard cutpoint (Fig. 6E and F). This geometric addition to the ROC plot, introduced by Biggerstaff (2), allows four pieces of information to be gleaned easily from a single plot (i.e., sensitivity, specificity, and the positive and negative likelihood ratios), allowing users to make a more informed decision.

Although not presented in this study, Linnet (14) showed how the posterior probabilities in the form of a scoring rule can be used for threshold selection. This approach shares a shortcoming associated with Youden's index in that the sensitivity and specificity are weighted equally. Nonetheless, a convenient feature of the scoring rule is the ability to calculate a score under a "no-test" scenario, that is, a test score can be calculated based only on the prior probabilities. In other words, what is the probability of correctly predicting disease without use of the predictor? This is not unlike comparing the AUROC with the area under the line of no discrimination, but the interpretation is more straight forward than comparing two areas, as discussed above.

TABLE 5. Properties of the management action thresholds (i.e., cutpoints) displayed in Figure 6 for two predictors of harvest scab at two damage thresholds

\begin{tabular}{|c|c|c|c|c|c|c|c|c|c|}
\hline$D_{\text {thresh }^{\mathrm{a}}}$ & Predictor $^{b}$ & Symbol $^{\mathrm{c}}$ & $P(D+)^{\mathrm{d}}$ & $\mathrm{CR}^{\mathrm{e}}$ & $f^{\prime}(\mathrm{FPP})^{\mathrm{f}}$ & FPPg & TPPh & $\mathrm{PV}(+)^{\mathrm{i}}$ & $P V(-)^{j}$ \\
\hline \multirow[t]{4}{*}{0.05} & \multirow[t]{4}{*}{$p_{\text {efr }}$} & $\nabla$ & 0.22 & 0.967 & 3.428 & 0.034 & 0.759 & 0.861 & 0.934 \\
\hline & & $\nabla$ & 0.22 & 0.333 & 1.182 & 0.079 & 0.846 & 0.752 & 0.955 \\
\hline & & O & 0.05 & 0.333 & 6.333 & 0.021 & 0.696 & 0.637 & 0.984 \\
\hline & & 0 & 0.75 & 0.333 & 0.111 & 0.441 & 0.966 & 0.868 & 0.846 \\
\hline \multirow[t]{4}{*}{0.05} & \multirow[t]{4}{*}{$p_{\mathrm{cls}}$} & $\Delta$ & 0.22 & 0.967 & 3.428 & 0.090 & 0.556 & 0.635 & 0.879 \\
\hline & & $\triangle$ & 0.22 & 0.333 & 1.182 & 0.203 & 0.787 & 0.522 & 0.930 \\
\hline & & $\nabla$ & 0.05 & 0.333 & 6.333 & 0.039 & 0.317 & 0.298 & 0.964 \\
\hline & & $\diamond$ & 0.75 & 0.333 & 0.111 & 0.709 & 0.978 & 0.805 & 0.812 \\
\hline \multirow[t]{4}{*}{0.10} & \multirow[t]{4}{*}{$p_{\text {efr }}$} & $\nabla$ & 0.22 & 0.983 & 3.485 & 0.030 & 0.774 & 0.878 & 0.938 \\
\hline & & $\nabla$ & 0.22 & 0.667 & 2.365 & 0.041 & 0.805 & 0.846 & 0.946 \\
\hline & & O & 0.05 & 0.667 & 12.67 & 0.010 & 0.646 & 0.766 & 0.982 \\
\hline & & $\bigcirc$ & 0.75 & 0.667 & 0.222 & 0.248 & 0.940 & 0.919 & 0.807 \\
\hline \multirow[t]{4}{*}{0.10} & \multirow[t]{4}{*}{$p_{\mathrm{cls}}$} & $\Delta$ & 0.22 & 0.983 & 3.485 & 0.048 & 0.478 & 0.736 & 0.866 \\
\hline & & $\triangle$ & 0.22 & 0.667 & 2.365 & 0.074 & 0.552 & 0.677 & 0.880 \\
\hline & & $\nabla$ & 0.05 & 0.667 & 12.67 & 0.009 & 0.238 & 0.573 & 0.961 \\
\hline & & $\diamond$ & 0.75 & 0.667 & 0.222 & 0.723 & 0.949 & 0.797 & 0.644 \\
\hline
\end{tabular}

a The harvest scab damage threshold is the incidence of scab on harvested fruit considered unacceptable and was used to classify data into cases and controls.

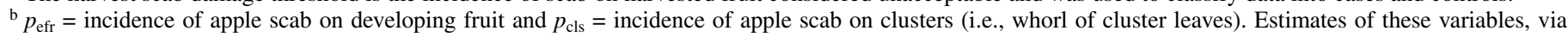
mid-season sampling, were used to predict the incidence of harvest scab.

c Symbols correspond to those displayed in Figure 6C to F.

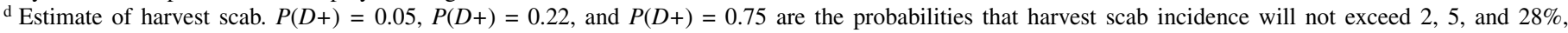
respectively. The probabilities were calculated over all treatments and untreated checks for the 5 years of data.

e The cost ratio as defined in equation 3 .

f Slope at the point on the ROC curve where the cost ratio is minimized for a specified $P(D+)$; LR(+)is equivalent to $f($ FPP) (false positive proportion) (equation 3 ).

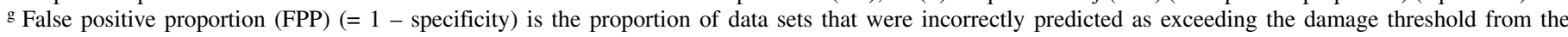
population of data sets that actually were below damage threshold (i.e., the number of controls incorrectly classified as a case).

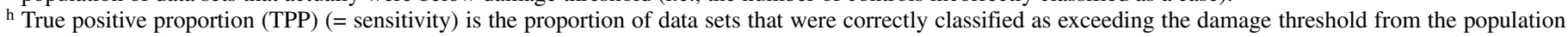
of data sets that actually were above the damage threshold (i.e., the number of cases that were correctly classified as such).

i Positive predictive value is the probability of harvest scab exceeding the damage threshold subsequent to the predictor exceeding the cutpoint.

j Negative predictive value is the probability of harvest scab not exceeding the damage threshold subsequent to the predictor exceeding the cutpoint. 
In conclusion, mid-season apple scab ratings can be used to predict whether the incidence of harvest scab will exceed a defined damage threshold. This prediction is intended to help growers decide the necessary intensity of their summer cover spray program. It should be kept in mind that growers relax the frequency of fungicide applications in their cover spray program primarily to reduce their costs and, to a lesser degree, to prevent the excessive use of fungicide. Naturally, the thought of reducing expenses can cloud their better judgment, causing growers to adjust their cover sprays without sufficient data to justify it. This research will help growers make the best decision by leading them to the best predictor of harvest scab and defining what incidence level of scab warrants an extended or reduced cover spray program. Unfortunately, good decisions do not always lead to good outcomes (22). But, do not let this be discouraging because the converse is more likely to be true, that is, good outcomes rarely result from bad decisions.

\section{LITERATURE CITED}

1. Bamber, D. 1975. The area above the ordinal dominance graph and the area below the receiver operating characteristic graph. J. Math. Psychol. 12:387-415.

2. Biggerstaff, B. J. 2000. Comparing diagnostic tests: A simple graphic using likelihood ratios. Stat. Med. 19:649-663.

3. Binns, M. R., Nyrop, J. P., and van der Werf, W. 2000. Sampling and Monitoring in Crop Protection: The Theoretical Basis for Developing Practical Decision Guides. CABI Publishing, Wallingford, UK.

4. DeLong, E. R., DeLong, D. M., and Clark-Pearson, D. L. 1988. Comparing the areas under two or more correlated receiver operating characteristic curves: A nonparametric approach. Biometrics 44:837-845.

5. Dewdney, M. M., Biggs, A. R., Lightner, G., and Turechek, W. W. 2002. MARYBLYT: Can there be improvements? (Abstr.) Phytopathology 92(suppl.):S19.

6. Dewdney, M. M, Biggs, A. R., and Turechek, W. W. 2003. A statistical comparison of MARYBLYT and Cougarblight using receiver operator characteristic curve analysis. (Abstr.) Phytopathology 93(suppl.):S20.

7. Fabre, F., Dedryver, C. A., Leterrier, J. L., and Plantegenest, M. 2003. Aphid abundance on cereals in autumn predicts yield losses caused by Barley yellow dwarf virus. Phytopathology 93:1217-1222.

8. Farrington, C. P. 1992. Estimating prevalence by group testing using generalized linear models. Stat. Med. 11:1591-1597.

9. Hanley, J. A., and McNeil, B. J. 1982. The meaning and use of the area under a receiver operating characteristic (ROC) curve. Radiology 143:2936.

10. Hilden, J. 1991. The are under the ROC curve and its competitors. Med. Decis. Making 11:95-101

11. Hughes, G., and Madden, L. V. 2003. Evaluating predictive models with application in regulatory policy for invasive weeds. Agric. Syst. 76:755774.

12. Hughes, G., McRoberts, N., and Burnett, F. J. 1999. Decision-making and diagnosis in disease management. Plant Pathol. 48:147-153.

13. Hughes, G., McRoberts, N., Madden, L. V., and Gottwald, T. R. 1997. Relationship between disease incidence at two levels in a spatial hierarchy. Phytopathology 87:542-550.

14. Linnet, K. 1988. A review on the methodology for assessing diagnostic tests. Clin. Chem. 34:1379-1386.
15. Lloyd, C. J. 2000. Regression models for convex ROC curves. Biometrics 56:862-867.

16. MacHardy, W. E. 1996. Chapter 28 in: Apple Scab: Biology, Epidemiology, and Management. The American Phytopathology Society, St. Paul, MN.

17. MacHardy, W. E., and Gadoury, D. M. 1989. A revision of Mills' criteria for predicting apple scab infection periods. Phytopathology 79:304-310.

18. Metz, C. E. 1978. Basic principles of ROC analysis. Nucl. Med. 8:283298.

19. Mills, W. D. 1944. Efficient use of sulfur dusts and sprays during rain to control apple scab. Cornell Univ. Ext. Bull. 630:1-4.

20. Murtaugh, P. A. 1996. The statistical evaluation of ecological indicators. Ecol. Appl. 6:132-139.

21. Pedigo, L. P., Hutchins, S. H., and Higley, L. G. 1986. Economic injury levels in theory and practice. Annu. Rev. Entomol. 31:341-368.

22. Ragsdale, C. T. 2004. Spreadsheet Modeling and Decision Analysis: A Practical Introduction to Management Science, 4th ed. South-Western, Mason, $\mathrm{OH}$

23. Schulzer, M. 1994. Diagnostic tests - A statistical review. Muscle Nerve 17:815-819.

24. Schwabe, W. F. S., Jones, A. L., and Jonker, J. P. 1984. Changes in the susceptibility of developing apple fruit to Venturia inaequalis. Phytopathology 74:118-121.

25. Tomerlin, J. R., and Jones, A. L. 1983. Development of apple scab on fruit in the orchard and during cold storage. Plant Dis. 67:147-150.

26. Turechek, W. W. 2004. Apple Diseases and their Management. Pages 1108 in: Diseases of Fruits and Vegetables and their Management. S. A. M. H. Naqvi, ed. Kluwer Publishers, Netherlands.

27. Turechek, W. W., and Madden, L. V. 1999. Spatial pattern analysis of strawberry leaf blight in perennial production systems. Phytopathology 89:421-433.

28. Turechek, W. W., and Madden, L. V. 2003. A generalized linear modeling approach for characterizing disease incidence in a spatial hierarchy. Phytopathology 93:458-466.

29. Twengström, E., Sigvald, R., Svensson, C., and Yuen, J. 1998. Forecasting sclerotinia stem rot in spring sown oilseed rape. Crop Prot. 17:405-411.

30. Wilcox, W. F., Burr, J. A., and Heidenreich, G. 1999. Evaluation of fungicide programs for control of scab and powdery mildew of apples. 1998. Fungic Nematic. Tests 54:31.

31. Wilcox, W. F., Burr, J. A., and Heidenreich, G. 2000. Evaluation of fungicide programs for control of scab and powdery mildew of apples. 1999. Fungic Nematic. Tests 55:36.

32. Wilcox, W. F., Burr, J. A., and Heidenreich, G. 2001. Evaluation of fungicide programs for control of scab and powdery mildew of apples, 2000. Fungic Nematic. Tests 56:PF37.

33. Wilcox, W. F., Burr, J. A., and Heidenreich, G. 2001. Evaluation of fungicide programs for control of scab and powdery mildew on 'Cortland' apples. 2000. Fungic Nematic. Tests 56:PF38.

34. Wilcox, W. F., Burr, J. A., Heidenreich, G., Smith, C., and Gadoury, D. M. 1998. Evaluation of solo fungicide products and nontraditional alternatives for control of scab and mildew. 1997. Fungic Nematic. Tests 53:35.

35. Wilcox, W. F., Burr, J. A., Heidenreich, G., Smith, C., and Gadoury, D. M. 1998. Evaluation of fungicide programs for control of scab and powdery mildew of apples. 1997. Fungic Nematic. Tests 53:37.

36. Wilcox, W. F., Burr, J. A., Smith, C., and Gadoury, D. M. 1997. Evaluation of fungicide programs for control of scab and powdery mildew of apples. 1996. Fungic Nematic. Tests 52:38.

37. Yuen, J., and Hughes, G. 2002. Bayesian analysis of plant disease prediction. Plant Pathol. 51:407-412.

38. Yuen, J., Twengström, E., and Sigvald, R. 1996. Calibration and verification of risk algorithms using logistic regression. Eur. J. Plant Pathol. 102:847-854. 